\title{
PERMUTATION GROUPS IN EUCLIDEAN RAMSEY THEORY
}

\author{
IGOR KŘİ̌̀
}

(Communicated by Andrew Odlyzko)

\begin{abstract}
A finite subset of a Euclidean space is called Ramsey if for each $k$ and each $k$-coloring of a sufficiently dimensional Euclidean space $E$ there is a monochromatic isometrical embedding from $F$ to $E$. We show that if $F$ has a transitive solvable group of isometries then it is Ramsey. In particular, regular polygons are Ramsey. We also show that regular polyhedra in $\mathbf{R}^{3}$ are Ramsey.
\end{abstract}

\section{INTRODUCTION}

The question of what configurations $F$ in a Euclidean space are Ramsey was first raised by Erdös, Graham, Montgomery, Rothschild, Spencer, and Straus [1]. They showed that in order for $F$ to be Ramsey, it must lie on a sphere. On the other hand, they showed that the property to be Ramsey is preserved by finite Cartesian products and thus that vertex sets of bricks are Ramsey. Frankl and Rödl [2] showed that all triangles and also infinitely many trapezoids are Ramsey. They asked whether there is a Ramsey pentagon.

In this paper we show that a configuration $F$ is Ramsey if it has a solvable transitive group of isometries or, more generally, if it has a transitive group of isometries which has a solvable subgroup with at most two orbits. In particular, regular polygons and regular polyhedra in $\mathbf{R}^{3}$ are Ramsey.

\section{Preliminaries}

2.1. Conventions and notations. $\mathbf{N}$ denotes the set of all natural numbers and $\mathbf{R}$ denotes the set of all real numbers. We make the identification

$$
n=\{0, \ldots, n-1\} .
$$

The notation $X \subset \subset Y$ for sets $X, Y$ means that $X$ is a subset of $Y$ and that $X$ is finite. The cardinality of a finite set $X$ is denoted by $|X|$. The image of a mapping $f: X \rightarrow Y$ is denoted by $\operatorname{Im} f$. If $R$ is a relation then $x R y$ means $(x, y) \in R$. If $E$ is an equivalence relation on $X$ then $X / E$ denotes the set of equivalence classes corresponding to $E$. For a set $A$, let $\left(\begin{array}{l}A \\ n\end{array}\right)$ denote

Received by the editors September 29, 1989.

1980 Mathematics Subject Classification (1985 Revision). Primary 05 C55.

Work on this paper was supported by NSF Grant DMS 8903132. 
the set of all subsets of $A$ of cardinality $n$. The symbol $\backslash$ denotes the settheoretical difference. For sets $A, B, A^{B}$ denotes the set of all mappings from $B$ to $A$. Thus, $A^{n}$ can be identified with the $n$th Cartesian power of $A$. For $a \in A^{n}$, we write $a=\left(a_{0}, \ldots, a_{n-1}\right)$. If $X \subset \subset \mathbf{R}, X=\left\{x_{1}, \ldots, x_{k}\right\}$ and $x_{1}<\cdots<x_{k}$, we write $X=\left\{x_{1}<\cdots<x_{k}\right\}$. On $\mathbf{R}^{n}$ we have a scalar product $x \cdot y=\sum_{i \in n} x_{i} \cdot y_{i}$, a norm $\|x\|=x \cdot x$ and the associated Euclidean metric. For $X \subseteq \mathbf{R}^{n}$ and $\lambda \in \mathbf{R}$ we put

$$
\lambda \cdot X=\{\lambda x \mid x \in X\} .
$$

2.2. The configurations. In this paper, a configuration $F$ is a finite subset of a Euclidean space $\mathbf{R}^{n}$. Thus, every configuration has a structure of a (finite) metric space. Thus, for configurations $F \subset \subset \mathbf{R}^{n}, H \subset \subset \mathbf{R}^{n}$ we have the notion of an isometry $\phi: F \rightarrow H \quad(a, 1-1$ correspondence preserving metric) and of an isometrical embedding $\phi: F \rightarrow \mathbf{R}^{n}$. To clean up the terminology, we present the following well-known fact:

2.2.1. Observation. Let $F \subset \subset \mathbf{R}^{m}$ be a configuration and let $\phi: F \rightarrow \mathbf{R}^{n}$ be an isometrical embedding. Further let $n \leq m$. Then there is a unique isometrical embedding $\psi: \mathbf{R}^{n} \rightarrow \mathbf{R}^{m}$ such that the following diagram commutes:

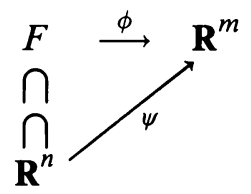

Proof. Let $F=\left\{x_{0}, \ldots, x_{k}\right\}$. Without loss of generality, the set $\left\{x_{i}-x_{k} \mid i \in k\right\}$ generates $\mathbf{R}^{n}$. Thus, without loss of generality, $\left\{x_{i}-x_{k} \mid i \in n\right\}$ is a basis of $\mathbf{R}^{n}$. Put $a_{i}=x_{i}-x_{k}, b_{i}=\phi\left(x_{i}\right)-\phi\left(x_{k}\right)$ and define $\psi$ by

$$
\psi\left(x_{k}+\sum_{i \in n} s_{i} a_{i}\right)=\phi\left(x_{k}\right)+\sum_{i \in n} s_{i} b_{i} .
$$

Since $\phi$ is an isometry, we have $\left\|a_{i}\right\|=\left\|b_{i}\right\|$ and $\left\|a_{i}-a_{j}\right\|=\left\|b_{i}-b_{j}\right\|$. Thus, $a_{i} \cdot a_{j}=b_{i} \cdot b_{j}$ and $\psi$ is an isometrical embedding.

2.3. The group actions. Let $X$ be a finite set, let $b: X \rightarrow X$ be a bijection and let a group $G$ act on $X$. We adopt the following notation:

$$
\begin{aligned}
& \operatorname{Orb}_{b}(x)=\left\{b^{n} x \mid n \in \mathbf{N}\right\}, \\
& \operatorname{Orb}_{G}(x)=\{g x \mid g \in G\} .
\end{aligned}
$$

These sets are called orbits of $x \in X$. The group $G$ is called transitive if, for some, $x \in X$ (equivalently, for each $x \in X$ ), $\operatorname{Orb}_{G}(x)=X$. A configuration $F$ is transitive if it has a transitive group of isometries. We also define the stabilizer

$$
\mathrm{St}_{G}(x)=\{g \in G \mid g x=x\} .
$$


Let $E_{G}$ be the equivalence relation on $X$ given by

$$
x E_{G} y \text { if and only if }(\exists g \in G)(g x=y) .
$$

On the other hand, if $E$ is an arbitrary equivalence relation on $X$, we say that $b$ respects $E$ if

$$
x E y \Rightarrow b x E b y .
$$

In that case, $b$ induces a bijection $X / E \rightarrow X / E$. By abuse of notation, we also denote it by $b$. Similarly, we say that $G$ respects $E$ if every $g \in G$ does. In that case $G$ acts on $X / E$. If $G$ respects $E$, we put

$$
\mathrm{St}_{G}(E)=\{g \in G \mid(\forall x \in X) g x E x\}=\{g \in G \mid(\forall z \in X / E) g z=z\} .
$$

Unlike $\mathrm{St}_{G}(x), \mathrm{St}_{G}(E)$ is always a normal subgroup of $G$. We put

$$
\begin{aligned}
& G / E=G / \mathrm{St}_{G}(E), \\
& \bigcup(E ; G)=\left\{(x, y) \in X^{2} \mid(\exists g \in G)(g x E y)\right\} .
\end{aligned}
$$

Choosing $n \in \mathbf{N}, x \in X$, we also put

$$
\bigcup(E ; x, b, n)=E \cup\left\{(y, z) \in X^{2} \mid(\exists i, j \in n)\left(\left(y E b^{i} x\right) \&\left(z E b^{j} x\right)\right)\right\} \text {. }
$$

2.3.1. Lemma. Let $G$ act on $X$ and let $H \subseteq G$ be a normal subgroup. Then:

$$
\begin{aligned}
& G \text { respects } E_{H}, \\
& G / E_{H} \text { is a factor group of } G / H, \text { and } \\
& \bigcup\left(E_{H} ; G\right)=E_{G} .
\end{aligned}
$$

Proof. Let $x E_{H} y$, i.e. $h x=y$. Then $(g h g)^{-1} g x=g y$, where $g h g^{-1} \in H$ since $H$ is normal. (2.3.1.1) is proved. By definition, $h \in H \Rightarrow h x E_{H} x$. Thus, $H \subseteq \mathrm{St}_{G}\left(E_{H}\right)$ and (2.3.1.2) is proved. Finally, $(\exists g \in G)\left(g x E_{H} y\right)$ if and only if $(\exists g \in G)(\exists h \in H)(h g x=y)$ if and only if $(\exists g \in G) g x=y$ if and only if $x E_{G} y$, proving (2.3.1.3).

2.4. The Ramsey properties. Consistently with [1], we adopt the following terminology. Let $F$ be a configuration, let $E$ be an equivalence relation on $F$ and let $s \in \mathbf{N}$. We say that $F$ is Ramsey (resp. E-Ramsey, resp. $s$-Ramsey) if for each $k \in \mathbf{N}$ there is an $m \in \mathbf{N}$ such that for each mapping $\sigma: \mathbf{R}^{n} \rightarrow k$ there is an isometrical embedding $\phi: F \rightarrow \mathbf{R}^{n}$ with the property that

$\sigma \phi$ is a constant resp. $x E y \Rightarrow \sigma \phi(x)=\sigma \phi(y)$, resp. $|\operatorname{Im} \sigma \phi| \leq s$.

2.4.1. Proposition. Let $F$ be an s-Ramsey configuration. Then there is an equivalence relation $E$ on $F$ such that $F$ is E-Ramsey and $|F / E| \leq s$.

Proof. Suppose that $F$ is $E$-Ramsey for no equivalence relation $E$ with $|F / E|$ $\leq s$. Then for each such relation $E$ there is a number $k_{E}$ such that for each $m \in \mathbf{N}$ there is a mapping $\sigma_{E}^{m}: \mathbf{R}^{m} \rightarrow k_{E}$ with the property that for no 
isometrical embedding $\phi: F \rightarrow \mathbf{R}^{m} \sigma_{E}^{m} \phi$ is constant on equivalence classes of $E$. Define

$$
\sigma^{m}: \mathbf{R}^{m} \rightarrow \prod_{|F / E| \leq s} k_{E}
$$

by

$$
\left(\sigma^{m}(x)\right)_{E}=\sigma_{E}^{m}(x) .
$$

Then there is no isometrical embedding $\phi: F \rightarrow \mathbf{R}^{m}$ such that

$$
\left|\operatorname{Im} \sigma^{m} \phi\right| \leq s \text {. }
$$

Indeed, put

$$
E=\left\{(x, y) \in F^{2} \mid \sigma^{m} \phi(x)=\sigma^{m} \phi(y)\right\}
$$

and suppose that $(2.4 .1 .1)$ holds. Then

$$
|F / E|=\left|\operatorname{Im} \sigma^{m} \phi\right| \leq s .
$$

Thus, for some $x, y \in F$, we have

$$
\begin{gathered}
x E y \\
\sigma_{E}^{m} \phi(x) \neq \sigma_{E}^{m} \phi(y) .
\end{gathered}
$$

However, (2.4.1.2) implies $\sigma^{m} \phi(x)=\sigma^{m} \phi(y)$ and thus contradicts (2.4.1.3).

\section{THE PRODUCT THEOREM}

3.1. Definition. Let $F_{1}, F_{2}$ be configurations and let $E_{i}$ be an equivalence relation on $F_{i}$. Then $F_{1} \times F_{2}$ (with the Euclidean product of the metrics) has the structure of a configuration. Moreover, it comes with an equivalence relation $E_{1} \times E_{2}$ given by

$$
\left(x_{1}, x_{2}\right)\left(E_{1} \times E_{2}\right)\left(y_{1}, y_{2}\right) \text { if and only if }\left(x_{1} E_{1} y_{1}\right) \&\left(x_{2} E_{2} y_{2}\right) \text {. }
$$

Analogously, we define a power $F^{n}$ of a configuration with a power $E^{n}$ of the equivalence relation.

The following result strengthens two theorems from [1].

3.2. The Product Theorem. Let $F_{1}, F_{2}$ be configurations and let $E_{i}$ be an equivalence relation on $F_{i}$ such that $F_{i}$ is $E_{i}$-Ramsey. Then $F_{1} \times F_{2}$ is $\left(E_{1} \times E_{2}\right)$ Ramsey.

Proof. Assume that for both $i \in\{1,2\}$ and for each $k \in \mathbf{N}$ there is an $N_{i}(k) \in$ $\mathbf{N}$ such that for each mapping

$$
\sigma: \mathbf{R}^{N_{i}(k)} \rightarrow k
$$

there is an isometrical embedding $\phi: F_{i} \rightarrow \mathbf{R}^{N_{i}(k)}$ with $x E_{i} y \Rightarrow \sigma \phi(x)=\sigma \phi(y)$. Put

$$
m_{1}=N_{1}\left(k^{\left|F_{2} / E_{2}\right|}\right)
$$


By an easy compactness argument, there is an $X \subset \subset \mathbf{R}^{m_{1}}$ such that for each mapping

$$
\sigma: X \rightarrow k^{\left|F_{2} / E_{2}\right|}
$$

there is an isometrical embedding $\phi: F_{1} \rightarrow X$ with $x E_{1} y \Rightarrow \sigma \phi(x)=\sigma \phi(y)$. Put $m_{2}=N_{2}\left(k^{|X|}\right)$. Now consider an arbitrary mapping

$$
\sigma: \mathbf{R}^{m_{1}+m_{2}} \rightarrow k \text {. }
$$

First define a mapping

$$
\sigma_{2}: \mathbf{R}^{m_{2}} \rightarrow k^{X}
$$

by putting

$$
\left(\sigma_{2}(y)\right)_{x}=\sigma\left(x_{0}, \ldots, x_{m_{1}-1}, y_{0}, \ldots, y_{m_{2}-1}\right) .
$$

By definition, there is an isometrical embedding

$$
\phi_{2}: F_{2} \rightarrow \mathbf{R}^{m_{2}}
$$

such that

$$
x E_{2} y \Rightarrow \sigma_{2} \phi_{2}(x)=\sigma_{2} \phi_{2}(y) .
$$

Now define a mapping

$$
\sigma_{1}: X \rightarrow k^{F_{2} / E_{2}}
$$

by

$$
\left(\sigma_{1}(x)\right)_{y}=\sigma\left(x_{0}, \ldots, x_{m_{1}-1}, z_{0}, \ldots, z_{m_{2}-1}\right),
$$

where $y \in F_{2} / E_{2}$ and $z \in y$. By (3.2.1), the choice of $z$ is immaterial. We conclude that here is an isometrical embedding $\phi_{1}: F_{1} \rightarrow X$ such that

$$
x E_{1} y \Rightarrow \sigma_{1} \phi_{1}(x)=\sigma_{1} \phi_{1}(y) .
$$

Now let $l: X \rightarrow \mathbf{R}^{m_{1}}$ be the inclusion. Then obviously

$$
\left(l \phi_{1} \times \phi_{2}\right): F_{1} \times F_{2} \rightarrow \mathbf{R}^{m_{1}+m_{2}}
$$

is an isometrical embedding, while, by (3.2.1), (3.2.2),

$$
x\left(E_{1} \times E_{2}\right) y \Rightarrow \sigma\left(l \phi_{1} \times \phi_{2}\right)(x)=\sigma\left(l \phi_{1} \times \phi_{2}\right)(y) .
$$

\subsection{Theorem. Any 2-Ramsey transitive configuration is Ramsey.}

Proof. Let $F$ be a 2-Ramsey configuration. By Proposition 2.4.1, there is an equivalence relation $E$ on $F$ such that $F$ is $E$-Ramsey and $|F / E| \leq 2$. Without loss of generality we may assume $|F / E|=2$. Let $G$ be a transitive group of isometries of $F$. Put

$$
\begin{gathered}
F / E=\left\{z_{1}, z_{2}\right\} \\
t=\left|z_{1}\right| \cdot\left|\mathrm{St}_{G}(x)\right| \quad \text { where } x \in F .
\end{gathered}
$$

Note that the choice of $x \in F$ in (3.3.2) is immaterial since $G$ is transitive. 
Fix a $k \in \mathbf{N}$. Now let $m \in \mathbf{N}$ satisfy the following statement (cf. [3]): For any mapping

$$
\tau:\left(\begin{array}{c}
m \\
t
\end{array}\right) \rightarrow k
$$

there is a subset $M \subseteq n$ such that

$$
|M|=|G|
$$

and

$$
\tau \mid\left(\begin{array}{c}
m \\
t
\end{array}\right) \text { is constant. }
$$

By Theorem 3.2, $F^{m}$ is $E^{m}$-Ramsey. Thus, there is an $N \in \mathbf{N}$ such that for each mapping $\sigma_{2}: \mathbf{R}^{N} \rightarrow k$ there is an isometrical embedding $\psi: F^{m} \rightarrow \mathbf{R}^{N}$ with the property that

$$
x E^{m} y \Rightarrow \sigma \psi(x)=\sigma \psi(y) .
$$

Choose $\bar{z}_{j} \in z_{j}$ and define, for $P \in\left(\begin{array}{c}m \\ t\end{array}\right), u(P) \in F^{m}$ by

$$
\begin{array}{ll}
(u(P))_{i}=\bar{z}_{1} & \text { if } i \in P, \\
(u(P))_{i}=\bar{z}_{2} & \text { if } i \in m \backslash P .
\end{array}
$$

Define, further,

$$
\tau:\left(\begin{array}{c}
m \\
t
\end{array}\right) \rightarrow k
$$

by

$$
\tau(P)=\sigma \psi(u(P)) .
$$

Choose an $M \subseteq n$ satisfying (3.3.3) and (3.3.4). Choose, further, a bijection $\imath: G \rightarrow M$ and define a mapping $\zeta: F \rightarrow F^{m}$ by

$$
\begin{aligned}
& (\zeta(x))_{i}=\bar{z}_{2} \quad \text { if } i \notin M, \\
& (\zeta(x))_{l(g)}=g x .
\end{aligned}
$$

Obviously, $\zeta$ enlarges distances by a constant factor of $(|G|)^{1 / 2}$. We shall prove

$$
\sigma \psi \zeta \text { is constant. }
$$

Indeed, for each $x \in F$, the set

$$
P_{x}=\left\{i \in m \mid(\zeta(x))_{i} \in z_{1}\right\}
$$

satisfies $P_{x} \subseteq M$ and $\left|P_{x}\right|=t$. (The latter statement is by (3.3.2) and the transitivity of $G$.) Now (3.3.6) follows from (3.3.4) and (3.3.5). We conclude that $(|G|)^{1 / 2} \cdot F$ is Ramsey and hence so is $F$.

Exactly in the same way we can prove the following more general result. 
3.4. Theorem. Let $F$ be a configuration and let $E_{1} \subseteq E_{2} \subseteq F$ be equivalence relations on $F$ such that $F$ is $E_{2}$-Ramsey, $\left|F / E_{2}\right| \leq 2$ and let $G$ be a group of isometries of $F$ which respects $E_{1}$. Then $F$ is $U\left(E_{1} ; G\right)$-Ramsey.

\section{THE MAIN RESULT}

4.1. Theorem. Let $F$ be an E-Ramsey configuration and let $b: F \rightarrow F$ be an isometry which respects $E$. Then, for each $z \in F$ and each $n \in \mathbf{N}, f$ is $\bigcup(E ; z, b, n)$-Ramsey.

Proof. An induction on $n$. Put

Assume $F$ is $\bar{E}$-Ramsey where $\bar{E}=\bigcup(E ; z, b, n-1)$. Choose a $k \in \mathbf{N}$.

$$
t=\left|\operatorname{Orb}_{b}(z)\right| \text {. }
$$

Let (cf. [3]) $m \in \mathbf{N}$ be such that for each

$$
\tau:\left(\begin{array}{c}
m \\
t-1
\end{array}\right) \rightarrow k^{n-1}
$$

there is an $M \subseteq m$ satisfying

$$
|M|=t
$$

and

$$
\tau \mid\left(\begin{array}{c}
M \\
t-1
\end{array}\right) \text { is constant. }
$$

Now, by Theorem 3.2, there is an $N \in \mathbf{N}$ such that for each mapping

$$
\sigma: \mathbf{R}^{N} \rightarrow k
$$

there is an isometrical embedding

$$
\psi: F^{m} \rightarrow \mathbf{R}^{N}
$$

satisfying

$$
x \bar{E}^{m} y \Rightarrow \sigma \psi(x)=\sigma \psi(y) .
$$

Now fix mappings (4.1.4), (4.1.5). For $\left\{p_{1}<\cdots<p_{t-1}\right\}=P \in\left(\begin{array}{c}m \\ t-1\end{array}\right)$ and for $i \in m-1$, define $u_{i}(P) \in F^{m}$ by

$$
\begin{aligned}
& \left(u_{i}(P)\right)_{j}=b^{i} \bar{z} \text { for } j \notin P \\
& \left(u_{i}(P)\right)_{p_{s}}=b^{i+s} z \text { for } 1 \leq s \leq t-1 .
\end{aligned}
$$

Define $\tau:\left(\begin{array}{c}m \\ t-1\end{array}\right) \rightarrow k^{n-1}$ by

$$
(\tau(P))_{i}=\sigma \psi u_{i}(P) \text { for } i \in n-1 .
$$

Let $M=\left\{p_{0}<\cdots<p_{t-1}\right\}$ satisfy (4.1.2) and (4.1.3). Define an embedding

$$
\bar{\phi}: F \rightarrow F^{m}
$$


by

$$
\begin{aligned}
& (\bar{\phi}(x))_{j}=z \text { for } j \notin M, \\
& (\bar{\phi}(x))_{p_{s}}=b^{s} z \text { for } 0 \leq s \leq t-1 .
\end{aligned}
$$

Obviously, $\bar{\phi}$ enlarges distances by a constant factor of $t^{1 / 2}$. Moreover, we have

$$
x E y \Rightarrow \bar{\phi}(x) E^{m} \bar{\phi}(y)
$$

(since $b$ respects $E$ ). Thus, by (4.1.6),

$$
x E y \Rightarrow \sigma \psi \bar{\phi}(x)=\sigma \psi \bar{\phi}(y) \text {. }
$$

We shall prove

$$
(\forall i \in\{1, \ldots, n-1\}) \quad\left(\sigma \psi \bar{\phi}\left(b^{i-1} z\right)=\left(\sigma \psi \bar{\phi}\left(b^{i} z\right)\right) .\right.
$$

Indeed, define, for $i \in n-1$ and $j \in n, u(i, j) \in F^{m}$ by

$$
\begin{aligned}
& (u(i, j))_{r}=b^{i} z \text { for } r \notin M \\
& (u(i, j))_{p_{s}}=b^{j+s} z \text { for } r \in M .
\end{aligned}
$$

We have, for $i \in\{1, \ldots, n-1\}$,

$$
\begin{aligned}
& \sigma \psi \bar{\phi}\left(b^{i-1} z\right) \quad \text { by (4.1.6) } \\
& =\sigma \psi(u(i-1, i-1)) \\
& =\sigma \psi\left(u_{i-1}(M \backslash\{\min M\})\right) \quad \text { by (4.1.3) } \\
& =\sigma \psi\left(u_{i-1}(M \backslash\{\max M\})\right) \quad \text { by (4.1.1), }(i-1)=i+(t-1) \bmod t \\
& \text { and (4.1.6) } \\
& =\sigma \psi(u(i-1, i)) \quad \text { by }(4.1 .6) \\
& =\sigma \psi \bar{\phi}\left(b^{i} z\right) \text {, }
\end{aligned}
$$

concluding the proof of (4.1.8). By (4.1.7) and (4.1.8), $t^{1 / 2} \cdot F$ is $t^{1 / 2}$ $\cup(E ; z, b, n)$-Ramsey and hence $F$ is $\bigcup(E ; z, b, n)$-Ramsey. The induction is complete.

4.2. Theorem. Let $F$ be an E-Ramsey configuration and let $G$ be a group of isometries of $F$ which respects $E$. Assume that $G / E$ is cyclic. Then $F$ is $\bigcup(E ; G)$-Ramsey.

Proof. The statement follows from Theorem 4.1 by induction on the number of orbits of $G / E$.

4.3. Theorem. Let $F$ be a configuration and let $G$ be a solvable group of isometries of $F$. Then $F$ is $E_{G}$-Ramsey.

Proof. The statement follows from Theorem 4.2 and Lemma 2.3.1.

4.4. Theorem. Any transitive configuration which has a solvable group of isometries with $\leq 2$ orbits is Ramsey.

Proof. The statement follows from Theorems 3.3 and 4.3. 

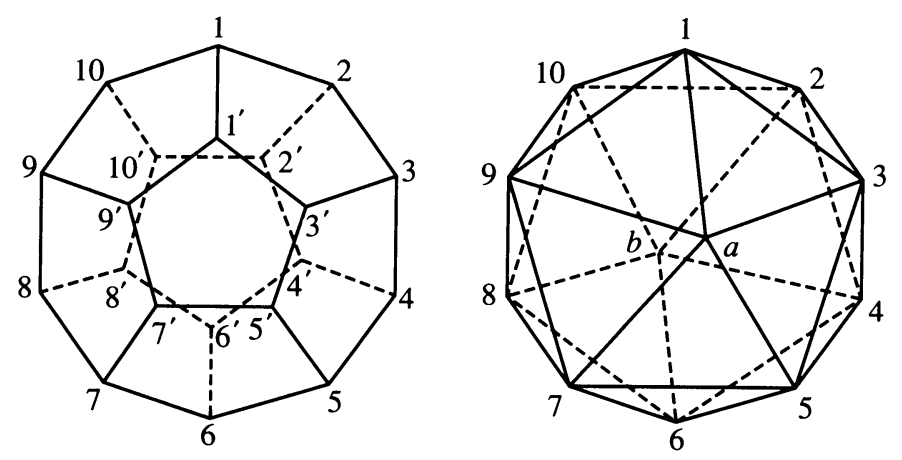

FIGURE 1

\subsection{Corollary. Vertex sets of regular polygons are Ramsey.}

4.6. Corollary. Vertex sets of regular polyhedra in $\mathbf{R}^{3}$ are Ramsey.

Proof. By the results of [1], we need only consider the cases of the dodecahedron and icosahedron. The reader may notice that these two polyhedra do not actually have solvable transitive groups of isometries. However, both of them have groups of isometries which have two orbits and are isomorphic to $Z_{10}$. If we label the vertices of the polyhedra as in Figure 1, the groups will be generated by the permutations corresponding to

$$
\begin{array}{ll}
(1,2,3,4,5,6,7,8,9,10) & \left(1^{\prime}, 2^{\prime}, 3^{\prime}, 4^{\prime}, 5^{\prime}, 6^{\prime}, 7^{\prime}, 8^{\prime}, 9^{\prime}, 10^{\prime}\right) \\
(1,2,3,4,5,6,7,8,9,10) & (a, b),
\end{array}
$$

respectively.

\section{ACKNOWLEDGMENT}

I am indebted to V. Rödl for valuable discussions.

\section{REFERENCES}

1. P. Erodös, R. L. Graham, P. Montgomery, B. L. Rothschild, J. Spencer, and E. G. Straus, Euclidean Ramsey theorems, J. Combin. Theory Ser. A 14 (1973), 341-363.

2. P. Frankl and V. Rödl, All triangles are Ramsey, Trans. Amer. Math. Soc. 2 (1986), 777779.

3. F. P. Ramsey, On a problem of formal logic, Proc. London Math. Soc. 30 (1930), 264-286.

Department of Mathematics, The University of Chicago, Chicago, Illinois 60637 